I N S T I T U T O

$\mathrm{DE}$

M E D I C I N A

T R O P I C A L

DE

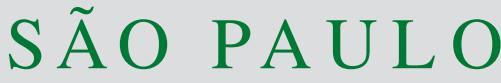

JOURNAL OF THE SÃO PAULO INSTITUTE OF TROPICAL MEDICINE

${ }^{1}$ Universidade Federal de Minas Gerais, Hospital das Clínicas, Hospital São Geraldo, Belo Horizonte, Minas Gerais, Brazil

${ }^{2}$ Instituto de Oftalmologia Cançado Trindade, Belo Horizonte, Minas Gerais, Brazil

${ }^{3}$ Universidade Federal de Minas Gerais, Faculdade de Medicina, Departamento de Oftalmologia e Otorrinolaringologia, Belo Horizonte, Minas Gerais, Brazil

${ }^{4}$ Universidade Federal de Minas Gerais, Faculdade de Medicina, Programa de Pós-Graduação em Ciências da Saúde - Infectologia e Medicina Tropical, Belo Horizonte, Minas Gerais, Brazil

${ }^{5}$ Universidade Federal de Minas Gerais, Faculdade de Medicina, Programa de Pós-Graduação em Ciências Aplicadas à Cirurgia e à Oftalmologia, Belo Horizonte, Minas Gerais, Brazil

${ }^{6}$ Instituto da Visão, Belo Horizonte, Minas Gerais, Brazil

Correspondence to: Daniel Vitor Vasconcelos-Santos Universidade Federal de Minas Gerais, Hospital das Clínicas, Hospital São Geraldo, Av. Alfredo Balena,190, sala 199, CEP 30130-100, Belo Horizonte, MG, Brazil

E-mail: dvitor@ufmg.br

Received: 21 July 2021

Accepted: 11 October 2021

\section{Cytomegalovirus as a possibly overlooked agent of hypertensive anterior uveitis and endotheliitis in immunocompetent patients in Brazil}

\author{
Caroline dos Reis', Bruno Avelar Miranda1, Aloysio Fellet da Cunha Afonso', \\ Leandro H. Malta e Cunha', Bruno Cançado Trindade ${ }^{(12}$, Daniel Vitor \\ Vasconcelos-Santos $1,3,4,5,6$
}

\section{ABSTRACT}

Cytomegalovirus (CMV) is a member of the Herpesviridae family, including viruses that are well-known agents of keratitis, anterior uveitis, scleritis and retinitis. CMV is usually associated with ocular diseases in immunosuppressed individuals, with a notable exception of hypertensive anterior uveitis with distinctive clinical features in immunocompetent patients. This syndrome was characterized in the last two decades in Europe and Southeast Asia, and then documented in the rest of world. Definitive diagnosis in these cases is usually made by Polymerase Chain Reaction (PCR) of the anterior chamber fluid. We report three immunocompetent Brazilian adults with history of multiple glaucomatocyclitic crises and presenting with chronic hypertensive anterior uveitis invariably with mild anterior chamber inflammation and characteristic scarce nummular keratic precipitates. CMV DNA was successfully amplified and detected in the aqueous humor of all patients. Corneal endothelial counts were significantly reduced in the involved eyes, with one patient developing bullous keratopathy. All patients were then treated with topical ganciclovir gel and corticosteroids, with subsequent control of the intraocular inflammation. CMV may represent an overlooked / underestimated etiology of hypertensive anterior uveitis that may progressively lead to endothelial dysfunction, culminating in bullous keratopathy. Management of patients is challenging, with the potential use of topical antivirals to decrease the number of relapses, and corticosteroids to control anterior uveitis / endotheliitis and to protect the corneal endothelium.

KEYWORDS: Cytomegalovirus. Anterior uveitis. Glaucomatocyclitic crisis. Hypertensive anterior uveitis.

\section{INTRODUCTION}

Cytomegalovirus (CMV) is a ubiquitous herpesvirus with seroprevalences ranging from 51.5-54.4\% in Western countries to $87-100 \%$ in Asia ${ }^{1}$. CMV does not usually cause ocular inflammation in immunocompetent individuals ${ }^{2}$. Infection of the eye is classically known to give rise to CMV retinitis in immunocompromised patients, especially those with AIDS and low CD4 counts ${ }^{3}$.

With the advent of the Polymerase Chain Reaction (PCR) to analyze ocular fluids, CMV-DNA has been increasingly identified in aqueous humor of immunocompetent patients with hypertensive anterior uveitis ${ }^{3,4}$. In these cases, clinical features are apparently distinctive, falling into the spectrum between Fuchs heterochromic iridocyclitis (FHI) and Posner-Schlossman syndrome (PSS) ${ }^{5}$. This etiological 
relationship is reinforced by the correlation between viral DNA quantification in the aqueous humor and the disease activity, and by a positive response to ganciclovir, administered either topically, intravenously or orally (valganciclovir) ${ }^{1,4}$.

Despite the documentation of this association worldwide 4 , to the best of our knowledge, CMV anterior uveitis in immunocompetent patients has not been reported in Brazil. Here, we characterize three cases of immunocompetent Brazilian adults with PCR-proven CMV chronic hypertensive anterior uveitis presenting with a history of recurrent glaucomatocyclitic crises and clinical features consistent with the other reports of chronic hypertensive anterior uveitis due to CMV described in the rest of the world.

\section{CASE REPORTS}

\section{Case 1}

A previously healthy 30 -year-old male had a history of multiple episodes of hypertensive anterior uveitis in his left eye (LE) since the age of 25 . He had undergone cataract surgery for a bilateral congenital cataract at the age of 3 (right eye, RE) and 18 (left eye, LE), despite an unremarkable gestational/neonatal history. Those uveitis attacks responded well to topical loteprednol, with rapid normalization of the intraocular pressure (IOP). Three years later, the patient developed hypertensive anterior uveitis in his RE. Signs and symptoms were similar to those of previous episodes in the LE. However, this time the response to loteprednol was poor, requiring the use of continuous $1 \%$ prednisolone drops. As they were tapered, the intraocular inflammation relapsed. Oral valacyclovir was also attempted, with no improvement in the number or the severity of relapses in RE.

Extensive uveitis workup was negative (including rheumatoid factor, antinuclear antibodies, serology for syphilis, angiotensin converting enzyme and HLA-B27). IgG for HSV (herpes simplex virus), VZV (varicella zoster virus), $\mathrm{CMV}$ and rubella viruses were positive, but with negative IgM for these viruses.

At this time, the best-corrected visual acuity (BCVA) was $20 / 20$ in the RE and 20/25 in the LE. Slit-lamp examination (SLE) of the RE revealed ciliary injection, mild corneal edema and a few nummular keratic precipitates (KPs) inferiorly, in addition to iris heterochromia (Figure 1). Minimal inflammation in the anterior chamber (AC), with $0.5+$ cell and $1+$ flare, could also be seen in the RE. Intraocular pressure (IOP) was $45 \mathrm{mmHg}$ in the RE. SLE and IOP of LE were within normal limits. The fundus examination was also normal in both eyes.

As a consequence of the clinical course with multiple episodes of hypertensive anterior uveitis, associated with minimal AC inflammation and sparse nummular KPs, in addition to the lack of response to valacyclovir, the possibility of CMV etiology was considered and an AC tap was performed in the RE. PCR results were positive for CMV, but negative for HSV, VZV, EBV (Epstein-Barr virus), and also for human herpesviruses 6, 7 and 8 .

The patient was then successfully treated with topical $0.15 \%$ ganciclovir gel (initially $5 x$ /day for 2 months, followed by $2 \mathrm{x} / \mathrm{day}$ ) and dexamethasone drops (once daily), for a period of one year. Remission was achieved for 2 years.

However, a new recurrence with mild intraocular inflammation, elevation of IOP and corneal edema led to the restart of the antiviral. The patient restarted the treatment with ganciclovir (at this time $2 \%$ drops $5 \mathrm{x} /$ day, in addition

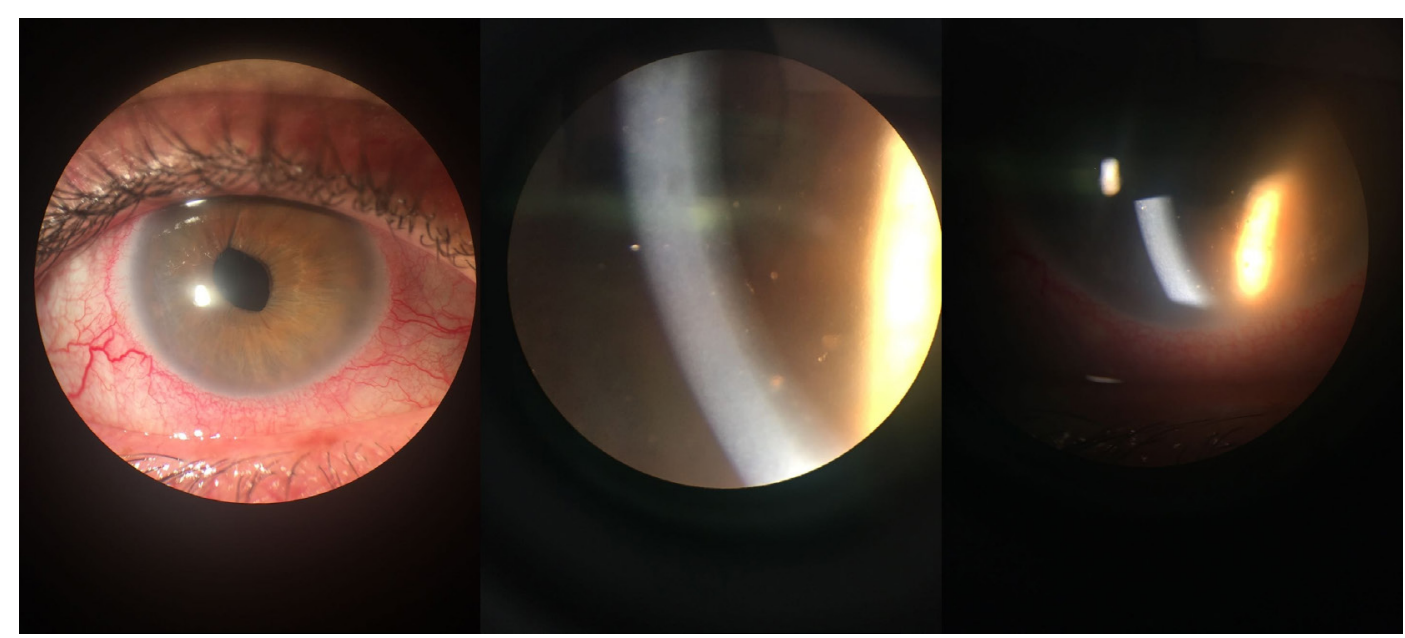

Figure 1 - Slit-lamp photographs of the right eye $(\mathrm{RE})$ in case 1, revealing ciliary injection, mild corneal edema and a few nummular keratic precipitates (KPs) inferiorly. There was a minimal inflammation in the anterior chamber, with $0.5+$ cell and $1+$ flare, that could also be seen in the RE. 
to $0.15 \%$ gel at bedtime) and topical dexamethasone, with subsequent resolution of the intraocular inflammation, and no recurrences after 3 years of further follow-up, now with topical $0.15 \%$ ganciclovir gel.

The specular microscopy revealed a significantly decreased endothelial cell count $\left(1,366\right.$ cells $/ \mathrm{mm}^{2}$ in the $\mathrm{RE}$, compared to 2,106 cells $/ \mathrm{mm}^{2}$ in the LE).

\section{Case 2}

A 45-year-old male had a history of multiple episodes of hypertensive anterior uveitis in the LE. He was otherwise healthy and had been treated with topical steroids and hypotensive drops. Extensive uveitis workup had been uneventful. However, since the last episode, he noticed that his vision did not come back to normal. On presentation, BCVA was 20/20 in the RE and 20/150 in the LE. SLE of the LE disclosed mild corneal edema with microbullae (bullous keratopathy), in addition to medium-sized coin-shaped KPs (Figure 2). Minimal AC reaction (1+cell and flare) was also noticed, as well significant posterior subcapsular cataract. No sectoral iris atrophy could be seen. IOP in the LE was elevated $(32 \mathrm{mmHg})$ and it was normal in the RE. The fundus examination was normal in both eyes, except for an increased cup-to-disc ratio of the left optic disc.

Due to the high suspicion of CMV etiology, AC tap was performed, being positive for CMV and negative for all other herpesviruses.

The patient was then treated with topical $2 \%$ ganciclovir eye drops $5 x /$ day and topical $0.15 \%$ ganciclovir gel at bedtime, in addition to $1 \%$ prednisolone drops. Intraocular inflammation and ocular hypertension in the LE subsided, and the patient is now waiting for endothelial keratoplasty and cataract surgery, with remission of the intraocular inflammation. Specular microscopy was not performed due to the corneal edema in the LE.

\section{Case 3}

An 89-year-old woman with systemic hypertension presented with a history of multiple episodes of hypertensive anterior uveitis in the RE after uneventful cataract surgery two years before. Extensive systemic investigations had been unrevealing, ruling out tuberculosis, syphilis and sarcoidosis, among other infections/noninfectious conditions. The ultrasound biomicroscopy showed a wellplaced intracapsular intraocular lens with no ciliary touch. Empiric treatment with topical and systemic corticosteroids led to the initial improvement, followed by recurrence of intraocular inflammation in the RE. Continuous use of timolol, dorzolamide and brimonidine drops in the RE was then necessary. At presentation, BCVA was 20/40 in the RE and 20/20 in the LE. Slit-lamp examination of the RE showed white medium-sized coin-shaped central/ paracentral keratic precipitates, connected by a fibrin network adhered to the corneal endothelium. The anterior chamber of the RE had 3+ cells and 1+ flare and the iris displayed focal atrophy inferonasally (Figure 3). A topic (inthe-bag) single-piece-intraocular lens (IOL) was also seen in both eyes. The anterior segment of the LE and the fundus examination of both eyes were otherwise unremarkable.

In face of the suspicion of an herpes virus etiology, an AC tap for PCR of aqueous humor, as well as serological tests for herpesviruses were performed. Results were positive for CMV DNA in aqueous humor, and for specific IgG anti-HSV, VZV and CMV in the serum. The specular microscopy revealed an endothelial cell count of 1,035 and 1,720 cells $/ \mathrm{mm}^{2}$ in the RE and the LE, respectively.

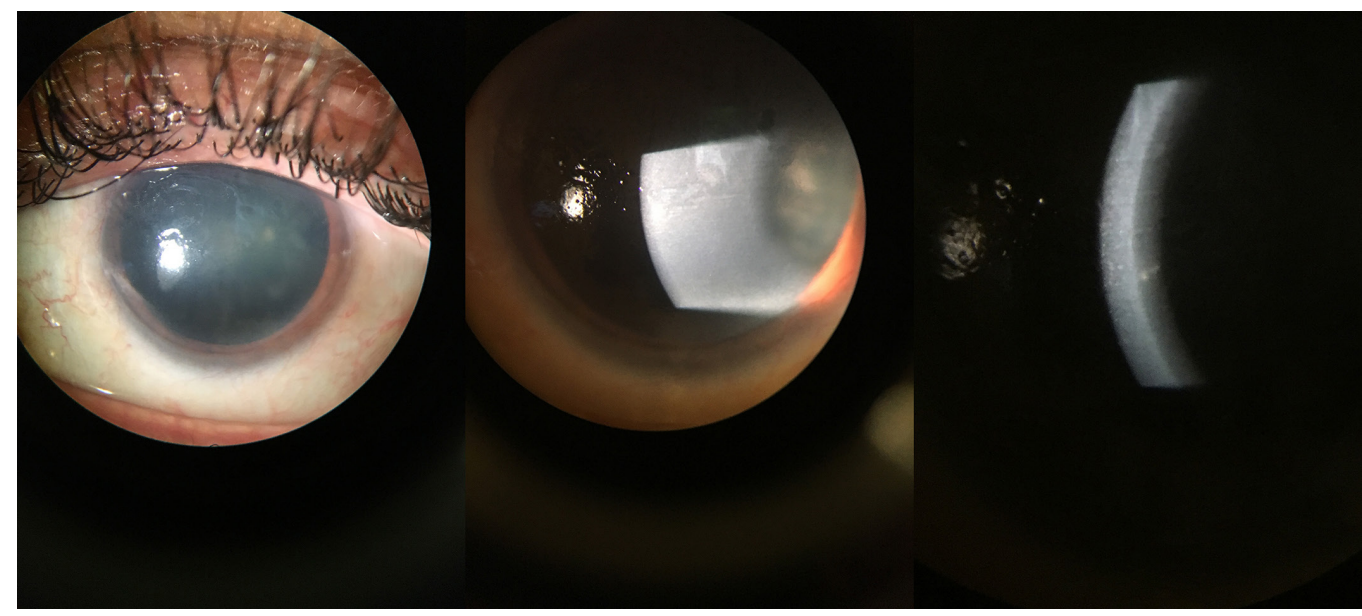

Figure 2 - Slit lamp photographs of the left eye (LE) of in case 2, disclosing mild corneal edema with microbullae (bullous keratopathy), in addition to medium-sized coin-shaped KPs. Minimal AC reaction (1+cell and flare) was observed, as well a significant posterior subcapsular cataract. No sectoral iris atrophy could be seen. 


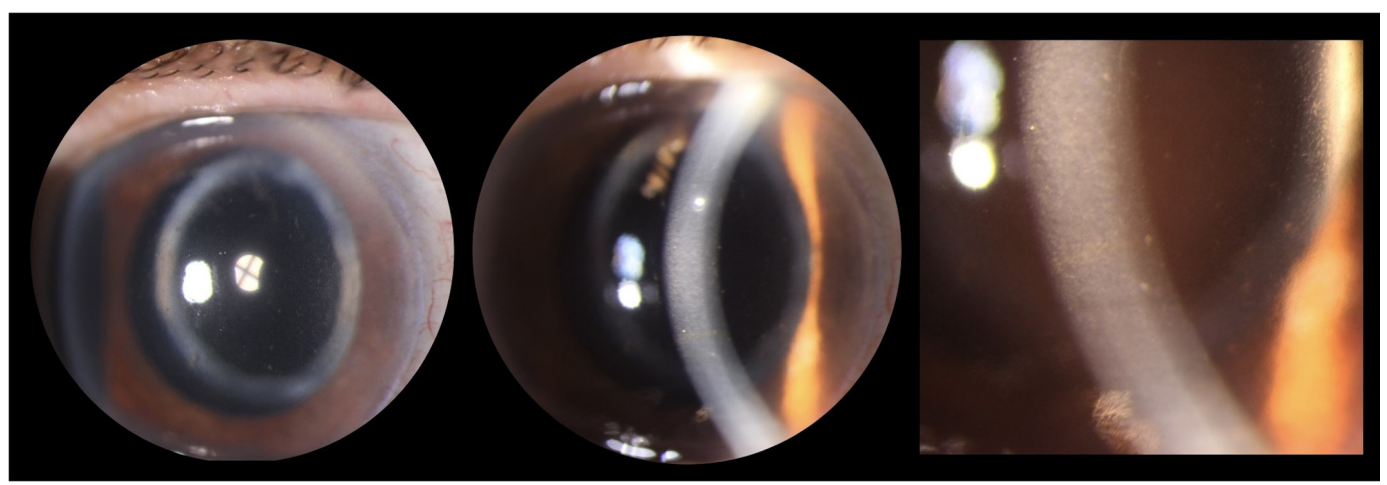

Figure 3 - Slit-lamp photographs of the right eye (RE) in case 3, revealing mild ciliary injection, inferonasal focal iris atrophy and a few nummular keratic precipitates (KPs)a t the bottom, associated with minimal fibrin deposition in the corneal endothelium.

Topical $2 \%$ ganciclovir eye drops $5 x /$ day and topical $0.15 \%$ ganciclovir gel at bedtime were then started, in addition to $1 \%$ prednisolone drops in a tapering regimen. The intraocular inflammation progressively improved, with no further crises of intraocular inflammation after six months of follow-up.

\section{DISCUSSION}

Viruses are being increasingly implicated in the etiology of otherwise presumed idiopathic uveitis syndromes, such as PSS and FHI. Various studies have supported the role of CMV as a pathogen in immunocompetent patients with hypertensive anterior uveitis, in different parts of the world, but surprisingly with no reports in Brazil. This may be explained by the fact that $\mathrm{AC}$ tap is not part of the routine investigation of uveitis in Brazil, and very few laboratories are used to deal with analysis of intraocular fluid in our country.

We reported three Brazilian adult patients with multiple episodes of hypertensive anterior uveitis, presenting with minimal signs of intraocular inflammation associated with the suggestive coin-shaped or nummular KPs. These cases fall within the spectrum of PSS-FHI-CMV anterior uveitis ${ }^{5}$. Case 1 showed chronic intraocular inflammation, but had some PSS-like crises and also had heterochromia. Case 2 arrived at our service at an advanced stage, but the history was consistent with PSS. Case 3 had a more recent diagnosis of intraocular inflammation and a shorter follow-up, with more suggestive signs and symptoms of a chronic anterior uveitis, despite having had multiple hypertensive PSS-like crises. This led us to consider the etiology of CMV and to collect and send aqueous humor for molecular diagnosis, which was positive for CMV and negative for all other herpesviruses. In addition, other important noninfectious and infectious etiologies were also excluded by extensive systemic investigations.

The diagnostic molecular assay consisted of a real-time PCR DNA microarray (Clart Entherpex kit [Genomica,
Coslada, Spain]), based on a viral, genome-specific amplification of a CMV fragment by multiplex PCR, followed by detection via hybridization with microorganismspecific binding probes on the low-density microarrays, for the simultaneous detection / identification of human herpesviruses (HSV-1, HSV-2, VZV, CMV, EBV, HHV-6, HHV-7, HHV-8), as well as enteroviruses ${ }^{6}$. Of note, the laboratory was instructed to handle and process aqueous humor samples similarly to cerebrospinal fluid samples. This is important because the total available volume of aqueous humor is limited and incorrect processing may result in insufficient material for analysis. This method does not allow the quantification of viral load in clinical samples. Therefore, it was not possible to correlate disease severity with viral load.

One patient had also developed posterior subcapsular cataract and bullous keratopathy in the infected eye. Topical antiviral therapy (combined with topical steroids) was immediately started after confirmation of the CMV infection, with subsequent control of intraocular inflammation and prevention of new relapses. In the first patient, a relapse of intraocular inflammation occurred one year after discontinuation of $0.15 \%$ ganciclovir. Because of recent evidence on low intraocular penetration of this preparation, ${ }^{7}$ this problem was solved with a more concentrated formulation ${ }^{8}$, combining $2 \%$ ganciclovir drops during the day with $0.15 \%$ ganciclovir gel at bedtime. This regimen was successfully employed for the second and for the third patients.

Losartan, a selective angiotensin II receptor antagonist, has recently been shown to inhibit the expression of TGF- $\beta 1$ and fibrogenic molecules in human trabecular meshwork (TM) cells, with the potential to decrease TM fibrosis in patients with CMV-induced hypertensive anterior uveitis ${ }^{9}$. However, losartan has not been tried in our patients. Treatment of anterior uveitis and endotheliitis caused by CMV is challenging, with no consensus on the drug, dosage, duration or modality of treatment. Even though topical ganciclovir seems to be a well-tolerated and less expensive 
therapy for CMV anterior uveitis, its efficacy may be inferior to systemic or intravitreal therapy ${ }^{1,10}$, depending on the concentration employed ${ }^{8}$. Limited availability of topical formulations is also a concern. Early suspicion of CMV as the etiology may allow clinicians to initiate CMV-specific therapy and to prevent sequelae, such as glaucoma, cataract and even endothelial cells $\operatorname{loss}^{2}$, the latter was observed in our patients.

Pathogenesis of anterior uveitis and endotheliitis due to CMV is largely unknown and it is surprising that it is not seen in immunosuppressed individuals ${ }^{1-5}$. Intraocular tissues may be indeed a site of CMV latency, as demonstrated in the murine model ${ }^{11}$. Local reactivation associated with "ocular immunoprivilege", i.e. the specifics of the immune response that occur within the eye, may be implicated, as seen in other intraocular infections caused by herpes viruses, with a likely prominent immunological component underlying intraocular inflammation ${ }^{5}$. CMV endotheliitis occurs more frequently in middle-aged and elderly men and usually present with corneal endotheliitis involving coin-shaped lesions, anterior uveitis and ocular hypertension ${ }^{12}$. Treatment of corneal endothelial decompensation may require a corneal graft. A timely identification of CMV as the causative agent of a corneal failure is particularly important, since early and thorough inflammation control may improve the graft survival ${ }^{13}$. Moreover, post-operative CMV reactivation may occur, and it may be clinically indistinguishable from an episode of endothelial graft rejection. Late graft failure can take place if specific treatment is delayed or not employed. CMV-specific medication such as ganciclovir and/or valganciclovir should be initiated before surgery and continued for at least six weeks after surgery. Therefore, CMV should be suspected as the causative agent of an endothelial decompensation in any unilateral hypertensive uveitis in immunocompetent patients, and an AC paracenthesis should be performed as soon as possible to confirm the findings ${ }^{13}$.

In conclusion, PCR-proven CMV hypertensive anterior uveitis may be found in Brazilian immunocompetent patients with history of recurrent glaucomatocyclitic crises and clinical features identical to those reported in other parts of the world. Aqueous humor PCR is the key to confirming the diagnosis. Management is challenging and aims to control the intraocular inflammation and minimize sequelae, including cataract, glaucoma and even endothelial failure.

\section{CONFLICT OF INTERESTS}

The authors report no conflict of interests related to this work.

\section{REFERENCES}

1. Chee SP, Jap A. Cytomegalovirus anterior uveitis: outcome of treatment. Br J Ophthalmol. 2010;94:1648-52.

2. Touhami S, Qu L, Angii M, Bojanova M, Touitou V, Lehoang P, et al. Cytomegalovirus anterior uveitis: clinical characteristics and long-term outcomes in a French series. Am J Ophthalmol. 2018;194:134-42.

3. Harada Y, Fukuda K, Nakahira A, Tada K, Sumi T, Fukushima A. Requirement of longer term antiviral therapy in patients with cytomegalovirus anterior uveitis with corneal endothelial cell damage. Clin Ophthalmol. 2018;12:1311.

4. Chee SP, Bacsal K, Jap A, Se-Thoe S, Cheng CL, Tan BH. Clinical features of cytomegalovirus anterior uveitis in immunocompetent patients. Am J Ophthalmol. 2008;145:83440.

5. Chan NS, Chee SP. Demystifying viral anterior uveitis: a review. Clin Exp Ophthalmol. 2019;47:320-33.

6. Leveque N, Van Haecke A, Renois F, Boutolleau D, Talmud D, Andreoletti L. Rapid virological diagnosis of central nervous system infections by use of a multiplex reverse transcriptionPCR DNA microarray. J Clin Microbiol. 2011;49:3874-79.

7. Waduthantri S, Zhou L, Chee SP. Intra-cameral level of ganciclovir gel, $0.15 \%$ following topical application for cytomegalovirus anterior segment infection: a pilot study. PloS One. 2018;13:e0191850.

8. Zhai RY, Xu H, Hong XM, Wang ZJ. Effect of $2 \%$ ganciclovir eye drops on cytomegalovirus positive Posner-Schlossman syndrome. Zhonghua Yan Ke Za Zhi. 2018;54:833-8.

9. Choi JA, Kim JE, Ju HH, Lee J, Jee D, Park CK, et al. The effects of losartan on cytomegalovirus infection in human trabecular meshwork cells. PLoS One. 2019;14:e0218471.

10. Hwang YS, Lin KK, Lee JS, Chang SH, Chen KJ, Lai CC, et al. Intravitreal loading injection of ganciclovir with or without adjunctive oral valganciclovir for cytomegalovirus anterior uveitis. Graefes Arch for Clin Exp Ophthalmol. 2010;248:2639.

11. Bale JF Jr, O’Neil ME, Hogan RN, Kern ER. Experimental murine cyto- megalovirus infection of ocular structures. Arch Ophthalmol. 1984;102:1214-9.

12. Koizumi N, Inatomi T, Suzuki T, Shiraishi A, Ohashi Y, Kandori $\mathrm{M}$, et al. Clinical features and management of cytomegalovirus corneal endotheliitis: analysis of 106 cases from the Japan corneal endotheliitis study. Br J Ophthalmol. 2015;99:54-8.

13. Fernández López E, Chan E. Descemet stripping automated endothelial keratoplasty outcomes in patients with cytomegalovirus endotheliitis. Cornea. 2017;36:108-12. 